\title{
Correction to: Bridging the Gaps: Multi Task Learning for Domain Transfer of Hate Speech Entection
}

\author{
Zeerak Talat, James Thorne, and Joachim Bingel
}

\section{Correction to:}

Chapter 3 in: J. Golbeck (ed.), Online Harassment, Human-Computer Interaction Series, https://doi.org/10.1007/978-3-319-78583-7_3

In the original version of the book, the misspelt co-author name "Zeerak Waseem" has been changed to read as "Zeerak Talat" in Chapter " 3 ". The erratum chapter and the book have been updated with the change. 